Correlation was made between NEWS2 and the total number of re-attendances within 28 days using regression analysis.

Results/Conclusions Simple linear regression analyses the influence of NEWS2 and the total number of re-attendances within 28 days and gives us the value of $\mathrm{R}^{2}$ as 0.007 , which suggests that 0.7 percent of variation of NEWS2 is attributable to regression (figure 1). The regression line is almost flat which tells us that there is no relationship between the two variables. NEWS2 alone is not a useful tool to predict reattendances to ED for patients presenting with COVID-19 symptoms.

\section{QUALITY IMPROVEMENT PROJECT - IMPROVING THE QUALITY OF CARE FOR HOMELESS PATIENTS PRESENTING TO THE HOMERTON UNIVERSITY HOSPITAL EMERGENCY DEPARTMENT 2019-20}

Phoebe Leung, Jane Chambers, Amber Morris, Kobbina Arthur, Fenella Prowse, Richard Crowson, William Niven. Homerton university hospital

\subsection{6/emj-2020-rcemabstracts. 15}

Aims/Objectives/Background Homelessness is on the rise in the UK. The problem identified specific to homeless patient care was clinician understanding of the homeless person's social needs to form an adequate discharge plan as well as completing their legal duty to refer such patients to the local housing authority.

Methods/Design This quality improvement project (QIP) aimed to reduce the reattendance rate of homeless patients presenting to the Homerton University Hospital (HUH) Emergency Department (ED) by 20\% from November 2019 to April 2020. This would be done by improving social history taking, signposting of patients to appropriate resources, and performing the legal duty to refer. Using the PDSA cycle method, interventions included a week of presentations to inform clinicians of the process measures; an advertising campaign; and a defined flowchart process for the duty to refer.

Results/Conclusions The QIP yielded the following results in terms of median baselines: social history taking $60 \%$ to $88 \%$, signposting to resources $30 \%$ to $67 \%$, and duty to refer 0 to $41 \%$. There was no change to the outcome measure of reattendance rate, maintained at $40 \%$ throughout the project and hence the QIP did not meet its SMART aim.

However this may have been the result of the decision to cut short data collection time due to the unprecedented COVID-19 pandemic which saw overall reduction in ED patient attendance. Most street homeless persons were put up in temporary hotels in the government funded scheme 'Everybody In', lockdown meant the hidden homeless should stay indoors, and a ban on court evictions has been extended until 23 August 2020.

Nonetheless, work to improve quality of care continued with a new pathway for safe discharge of homeless patients with suspected COVID-19. Planning ahead for post pandemic times has brought about a new standard operating procedure, which will ensure sustainability of the QIP.

\section{NHS 24 TRIAGE OF THE YOUNG ADULT WITH CHEST PAIN: AN ANALYSIS OF PATIENT CONTACTS IN UNSCHEDULED CARE}

${ }^{1}$ Peter Hodgins, ${ }^{2}$ Bruce Guthrie, ${ }^{2}$ Megan McMinn. ' Usher Institute, University of Edinburgh; ${ }^{2}$ University of Edinburgh

\subsection{6/emj-2020-rcemabstracts. 16}

Aims/Objectives/Background Use of unscheduled care is increasing worldwide. In the UK access to services for symptoms not thought to be life-threatening is through a single telephone advice and triage service (NHS 24 in Scotland). Adults under 35 account for the largest cohort contacting this

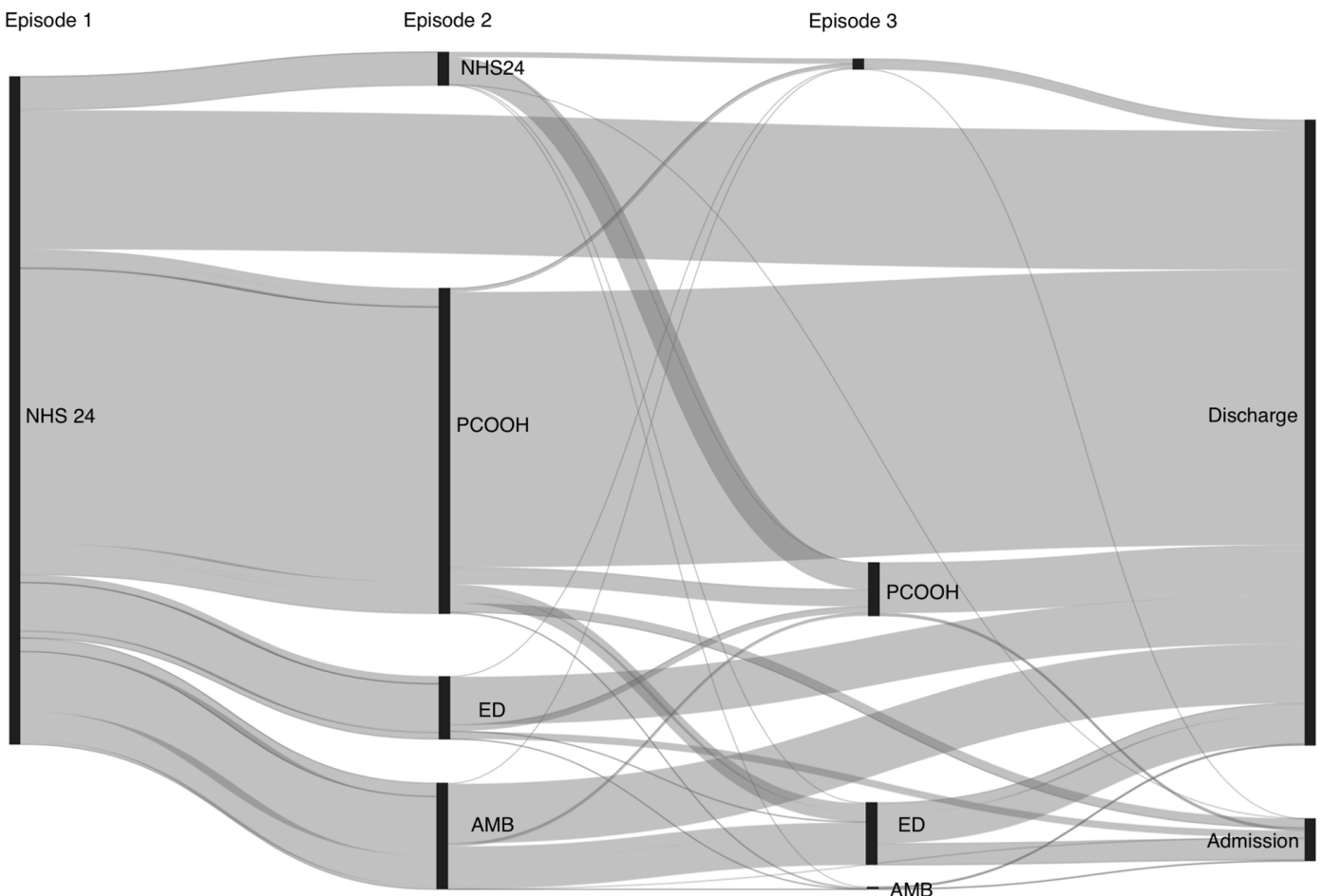


service. This study aimed to examine pathways of care in this population to inform areas for improvement to the triage process.

Methods/Design A retrospective, population study using the Scotland-wide Urgent Care Data Mart (UCD) 2015 to 2017. The UCD links data between unscheduled care services to form Continuous Urgent Care Pathways (CUPs). CUPs for those aged 15-34 years, whose initial contact was with NHS 24 and with 'chest pain' recorded as the call reason. Two outcomes were examined: admission to hospital, and admission to hospital with a 'time-critical diagnosis' defined as an admission with a discharge diagnosis of a condition that required urgent treatment.

Results/Conclusions A total of 102,822 CUPs initiated by a call to NHS24 with chest pain as the call reason in the period studied. $35.1 \%$ of calls were made by people living in the most deprived $20 \%$ of postcodes. The most common CUP pathway accounted for $37.6 \%$ and was an NHS 24 call followed by an attendance at Primary Care Out of Hours. $25.4 \%$ of calls were considered not to represent an urgent care need. The most frequent ICD-10 code was 'chest pain, unspecified', representing $7.2 \%$ of cases with an ICD-10 code, followed by 'asthma, unspecified' at 7.1\%. All NHS24 dispositions were strongly associated with increased odds of admission compared to self-care advice. Home-visits were associated with the greatest odds of time-critical diagnosis.

Chest pain is a symptom of concern for young people. Use of telephone triage is effective. 9060 CUPs result in a hospital admission, although many end with a non-specific diagnosis.

\section{WHAT ARE THE PRIORITIES FOR OLDER ADULTS ATTENDING THE ED? FINDINGS FROM A MULTIPLE STAKEHOLDER GROUP CONSENSUS MEETING}

${ }^{1}$ Blair Graham, ${ }^{1}$ Ruth Endacott, ${ }^{2} J a s o n$ E Smith, ${ }^{2}$ Ffion Barham, ${ }^{1} J o s$ M Latour. ${ }^{1}$ University of Plymouth: ${ }^{2}$ University Hospitals Plymouth NHS Trust

10.1136/emj-2020-rcemabstracts. 17

Aims/Objectives/Background Patient Reported Experience \& Outcome Measures (PREMs/PROMs) are not yet well established in emergency care. This study aimed to determine priorities for older adults attending the ED, to inform item inclusion for a new PREM/PROM.

Methods/Design One hundred thirty-five priority statements, linked to suggested PREM/PROM items, were derived from the literature, patient interviews $(n=24)$ and staff focus groups $(n=7)$ (October 2018- April 2019). All statements had a Flesch-Kincaid Reading Ease score $>70$. A one-day consensus meeting was held, December 2019.

Invitations were issued via social media, patient/public involvement groups and charities. Following focus groups to assess interpretability, nominal group technique was used to prioritise statements. Individuals scored statements from 1 (least important) to 9 (most important). Statements with a median score of $\geq 6.5$ were 'critically important', 3.5 to 6 'important, not critical', and $\leq 3$ 'less important'. Inter-rater agreement was assessed using mean absolute deviation from the median (MADM). 'Critically important' or 'less important' statements with MADM $<50 \%$ were automatically included or excluded, respectively. Statements deemed 'important, not critical', or with MADM $>50 \%$ underwent further voting.
Remaining statements underwent dichotomous voting. A $\geq 70 \%$ favourable majority was required for inclusion.

Results/Conclusions Twenty-nine participants attended. Average age was 65.6 years (R32-78). Experiences of emergency care were as a patient $(\mathrm{n}=16(55.2 \%))$, accompanying person $(n=11(37.9 \%))$, third-sector representative $(n=14(48.2 \%))$ and/ or clinician $(n=7(27.6 \%))$.

Initial prioritisation yielded 71 statements for inclusion. Further voting led to the inclusion of another 31 statements (102 total).

Statements relating to care delivery, communication and emotional needs ranked as more important overall. Statements related to waiting were considered less important (see supplementary file).

Participants reported having 'adequate say' during the meeting (76.9\% Strongly Agree).

Conclusion This meeting established priorities for older adults attending the ED. The low priority assigned to waiting confounds some previous findings. Further item reduction is planned to create a final PREM/PROM for older adults.

\section{COVID-19 RCEM RESEARCH SUMMARIES: TAKING THE BURDEN OFF BUSY CLINICIANS}

${ }^{1}$ Charles Reynard, ${ }^{2}$ Anisa Jafar, ${ }^{3}$ Govind Oliver, ${ }^{4}$ Daniel Darbyshire, ${ }^{5}$ Gabrielle Prager, ${ }^{1}$ Mina Naguib, ${ }^{1}$ Simon Carley. ${ }^{1}$ Central Manchester University Hospitals NHS Foundation Trust; ${ }^{2}$ University of Manchester; ${ }^{3}$ Royal Preston Hospital; ${ }^{4}$ Lancaster University; ${ }^{5}$ Royal Bolton Hospital

\subsection{6/emj-2020-rcemabstracts. 18}

Aims/Objectives/Background Entering lockdown on 23rd March 2020 due to the COVID-19 pandemic marked an unprecedented period for healthcare evidence. An exponential increase in published work, pre-prints, guidelines, online information portals and more, has been overwhelming especially when combined with the ever-changing local emergency department responses to COVID-19. Many research projects were either suspended in favour of clinical work or re-routed into pandemic-oriented studies. All the while, the gap between clinical providers and a mountain of information was growing. Our team developed a strategy to deliver the most pertinent evidence to those working in emergency medicine, taking some stress out this aspect of COVID-19 working.

Methods/Design Each week a search was conducted using PubMed of everything produced in the previous 7 days. The number of titles varied from approximately 800 to 2500. A 3 to 5 person team distilled titles and then reviewed abstracts for papers of importance and relevance to emergency medicine. Relevant and high impact journals were individually searched over the same time period. Summaries of the shortlisted papers were produced and the weekly editorial team selected 5 for inclusion in the weekly RCEM Top 5 and others were combined for extra reading as part of a 2-3 weekly 'Director's Cut'.

Results/Conclusions The RCEM Top 5 (at time of writing) has been run for 13 weeks. The summaries themselves were accessed by between 3000 and 6000 RCEM members/fellows each week. The work has also fed into online journal clubs and blogs (combined views of over 30,000) and has attracted interest from wider colleagues nationally and internationally to both join and even replicate the approach to other relevant areas. The positive feedback is best summarised in the following quote: 'when I'm too mentally overcooked to do any 